\title{
NRAS and BRAF mutation frequency in primary oral mucosal melanoma
}

\author{
ROSARIO RIVERA BUERY ${ }^{1}$, CHONG HUAT SIAR ${ }^{3}$, NAOKI KATASE ${ }^{2}$, MEHMET GUNDUZ MU,5 $^{4,5}$ \\ MATHIEU LEFEUVRE ${ }^{2}$, MASAE FUJII $^{2}$, MASAHISA INOUE ${ }^{1}$, KOJUN SETSU $^{1}$ and HITOSHI NAGATSUKA ${ }^{2}$ \\ ${ }^{1}$ Laboratories for Structure and Function Research, Faculty of Pharmaceutical Science, Tokushima Bunri University, \\ Tokushima; ${ }^{2}$ Department of Oral Pathology and Medicine, Graduate School of Medicine, Dentistry and Pharmaceutical \\ Sciences, Okayama University, Okayama, Japan; ${ }^{3}$ Department of Oral Pathology, Oral Medicine and Periodontology, \\ Faculty of Dentistry, University of Malaya, Kuala Lumpur, Malaysia; Departments of ${ }^{4}$ Otolaryngology \\ and ${ }^{5}$ Medical Genetics, Faculty of Medicine, Fatih University, Ankara, Turkey
}

Received April 7, 2011; Accepted May 9, 2011

DOI: 10.3892/or.2011.1385

\begin{abstract}
Oral mucosal melanoma (OMM) is a fatal sarcoma of unknown etiology. Histological morphology and genetic events are distinct from those of its cutaneous counterpart. Mutation and up-regulation of c-kit has been identified in OMM which may activate downstream molecules such as RAS and RAF. These molecules are involved in the mitogenactivated protein kinase (MAPK) pathway leading to tremendous cell proliferation and survival. NRAS and BRAF mutation and protein expression have been studied in other melanoma subtypes. The purpose of this study was to determine RAS protein expression and NRAS and BRAF mutation in 18 primary OMM cases using immunohistochemistry and mutation analysis. Results showed that RAS is intensely expressed in both in situ and invasive OMMs. However, NRAS mutation was only observed in $2 / 15$ polymerase chain reaction (PCR) amplified cases both of which were silent mutations. On the other hand, BRAF missense mutations were observed only in $1 / 15$ cases with PCR amplification. NRAS and BRAF mutations were independent from previously reported c-kit mutations. The classical V600E BRAF mutation was not found; instead a novel V600L was observed suggesting that the oncogenic event in OMM is different from that in skin melanoma. The low frequency of NRAS and BRAF mutations indicate that these genes are not common, but probable events in OMM pathogenesis, most likely independent of c-kit mutation.
\end{abstract}

Correspondence to: Dr Rosario R. Buery, Department of Oral Pathology and Medicine, Graduate School of Medicine, Dentistry and Pharmaceutical Sciences, Okayama University, 2-5-1 Shikata-cho, Okayama, 700-8525, Japan

E-mail: rsrivera@rocketmail.com

Key words: oral mucosal melanoma, NRAS, BRAF, mutation, immunohistochemistry

\section{Introduction}

Oral mucosal melanoma (OMM) is a malignant tumor in the oral cavity characterized by adjoining proliferation of atypical melanocytes and alteration of their normal functions. Although $\mathrm{OMM}$ is a rare tumor observed in $0.5 \%$ of oral malignancies and $0.2-8 \%$ of all melanomas, it has an aggressive behavior with poor prognosis $(1,2)$. Precursor lesions have not been clearly elucidated but the onset of atypical melanocytic proliferation may be the earliest indication of its development $(1,3)$. OMM based on histological examination can be classified as in situ, invasive and the combination of both, the latter being the most commonly observed $(1,4)$.

Mitogen-activated protein kinase (MAPK) is the most common pathway described in oncogenic events during the progression of melanoma (5-8). One of the molecules that participate in this signal transduction cascade is RAS encoded by the RAS gene consisting of HRAS, KRAS and NRAS. Another molecule that leads to the activation of MAPK is RAF consisting of ARAF, BRAF and CRAF. Frequent mutations in NRAS and BRAF have been observed in cutaneous melanoma (9-11). The MAPK pathway together with the phosphoinositide 3-kinase cascade (PI3K) can be triggered by activation of c-kit leading to the recruitment of signaling proteins involved in tremendous cell proliferation and survival (12). Mutations in c-kit have been identified in mucosal melanomas rendering c-kit as a promising molecular target (13-15).

NRAS and BRAF mutations have been reported in subsets of mucosal melanomas, but most reports focused on combined mucosal sites (9,16-19). Most reports have claimed that NRAS and BRAF mutations are infrequent, justifying that mucosal melanoma is distinct from its cutaneous counterpart. However, a frequent NRAS mutation was reported in esophageal melanoma (20).

Thus far, therapeutics concerning OMM have not been clearly elucidated. The study aimed to determine RAS protein expression and the incidence of NRAS and BRAF mutations in OMM. The study also compared the NRAS and BRAF mutations to previous investigations on c-kit mutations (13). 
Table I. NRAS primers.

$\begin{array}{ll}\text { Exon } 1 & \text { Forward: 5'-ATGTGGCTCGCCAATTAACC-3' } \\ & \text { Reverse: 5'-CTGGGCCTCACCTCTATGGT-3' } \\ \text { Exon } 2 & \text { Forward: 5'-CACACCCCCAGGATTCTTAC-3' } \\ & \text { Reverse: 5'-GCTCCTAGTACCTGTAGAGG-3' }\end{array}$

Table II. BRAF primers.

\begin{aligned} & Exon 11 Forward: 5'-TCCCTCTCAGGCATAAGGTAA-3' \\ & Reverse: 5'-CGAACAGTGAATATTTCCTTTGAT-3' \\ & Exon 15 $\begin{array}{l}\text { Forward: 5'-TCATAATGCTTGCTCTGATAGGA-3' } \\ \text { Reverse: 5'-GGCCAAAAATTTAATCAGTGGA-3' }\end{array} \\ &$\hline\end{aligned}

\section{Materials and methods}

Tissue samples embedded in paraffin blocks. A total of 18 cases of primary OMM from the Okayama University Graduate School of Medicine, Dentistry and Pharmaceutical Sciences, embedded in paraffin blocks were retrospectively analyzed (4). The Institutional Review Board of the University approved the study. The cases were histologically classified according to the Western Society of Teachers in Oral Pathology classification (1). The oral melanotic macule was used as a negative control.

Immonuhistochemistry. Sections of $3 \mu \mathrm{m}$ were cut and prepared for immunohistochemistry. Tissue sections were deparaffinized and blocked for endogenous peroxidase using $0.3 \%$ hydrogen peroxide in methanol for $30 \mathrm{~min}$ and washed with Trisbuffered saline solution. Antigen retrieval was carried out by immersing the slides in citrate buffer ( $\mathrm{pH}$ 6.0) placed in a pressure cooker for $15 \mathrm{~min}$. The slides were covered with Vectastain Avidin-Biotin complex (mouse, Vector Laboratories, Burlingame, CA, USA) for 15 min followed by 1:20 dilution of a Pan-RAS antibody (OSI Pharmaceuticals, NY, USA) and incubated overnight at $4^{\circ} \mathrm{C}$. Antigenic sites were detected with the ready-to-use 3-amino-ethylcarbazole (AEC) substrate chromogen (DakoCytomation, Carpinteria, CA, USA).

Semi-quantitative analysis of immunohistochemical staining was performed as previously described (13). Briefly, negative (-) means no immunoreaction; focal $( \pm)$ means $<20 \%$ melanoma cells are positive with weak staining degree; moderate $(+)$ means $20-50 \%$ melanoma cells are positive with strong intensity; and intense $(++)$ means $>50 \%$ of melanoma cells are positive with strong intensity.

Mutation analysis. Genomic DNA extraction was performed on sections from paraffin blocks using the Dexpat kit (Takara Bio, Shiga, Japan) and the High Pure PCR Template kit (Roche Applied Science, Tokyo, Japan) following the manufacturer's protocol. Polymerase chain reaction (PCR) amplified exons 1 and 2 for NRAS and 11 and 15 for BRAF using forward and reverse primers [NC_000007.13 (NRAS) and NC_000001.10 (BRAF), http://www.ncbi.nlm.nih.gov/gene] (Tables I and II). PCR cycling conditions include initial denaturation at $94^{\circ} \mathrm{C}$
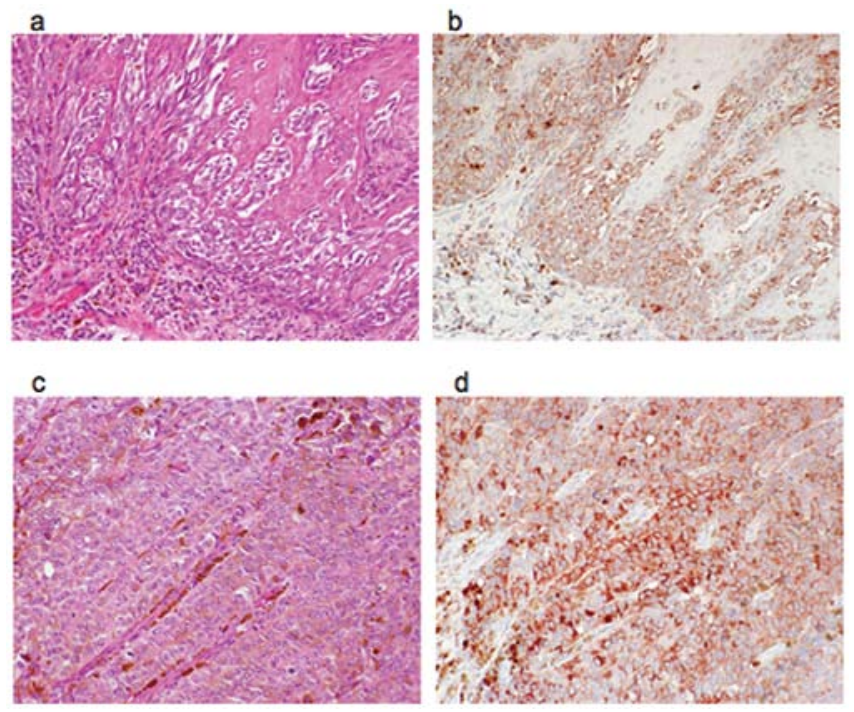

Figure 1. RAS protein expression. (a and c) H\&E of in situ and invasive OMM respectively. (b and d) Intense RAS expressions in in situ and invasive OMM, respectively (x20).

for $3 \mathrm{~min}, 45$ cycles of denaturation at $94^{\circ} \mathrm{C}$ for $30 \mathrm{sec}$, annealing at $60^{\circ} \mathrm{C}$ for NRAS and $54^{\circ} \mathrm{C}$ for BRAF for $1 \mathrm{~min}$, extension at $72^{\circ} \mathrm{C}$ for $1 \mathrm{~min}$ and a final extension at $72^{\circ} \mathrm{C}$ for $7 \mathrm{~min}$. PCR products were purified with the Geneclean III kit (QBiogene, USA) or treated with ExoSAP-IT (USB, OH, USA) before sequence-specific PCR. New sets of PCR products were subjected to single strand confirmation polymorphism (SSCP) where aberrant bands were cut, and subjected to PCR followed by ExoSAP-IT treatment and sequence-specific PCR.

Direct sequencing was performed using the Big Dye Terminator v1.1 Cycle Sequencing kit (Applied Biosystems, CA, USA). Sequence reactions were purified with methanol and EDTA precipitation and analyzed on the 3130xl ABI Prism Genetic Analyzer (Applied Biosystems, Hitachi, Japan). Repeat analysis of samples with mutations were carried out using independent DNA for confirmation purpose.

Results of RAS protein expression and NRAS and BRAF mutation analyses were compared to previously reported c-kit mutation and protein expression (13).

\section{Results}

Immunohistochemistry. Normal melanocytes in the oral melanotic macule were negative for the RAS protein (data not shown). On the other hand, tumor cells in OMM intensely expressed RAS. The intense expression was noted both in situ and invasive OMM. A total of 16/18 (89\%) cases expressed RAS protein (Fig. 1).

Mutation analysis. Frequent aberrant bands were observed in SSCP analysis of NRAS (Fig. 2a, arrows). However, when reactions were sequenced, only $2 / 15$ cases with PCR amplification $(13 \%)$ had point mutations. For instance, a nucleotide $\mathrm{G} \rightarrow \mathrm{A}$ change was observed, but there was no change in the amino acid (Fig. 2b).

Numerous aberrant bands were likewise observed in SSCP analysis of BRAF (Fig. 3a, arrows). However, only 1/15 cases 
Table III. Comparison of c-kit, NRAS and BRAF mutations in OMM.

\begin{tabular}{|c|c|c|c|c|c|c|c|}
\hline \multirow[b]{2}{*}{ Case no. } & \multicolumn{2}{|c|}{ c-Kit protein } & \multirow[b]{2}{*}{ c-Kit } & \multicolumn{2}{|c|}{ RAS protein } & \multirow[b]{2}{*}{ NRAS } & \multirow[b]{2}{*}{ BRAF } \\
\hline & In situ & Invasive & & In situ & Invasive & & \\
\hline 3 & NA & \pm & WT & NA & ++ & K166K & WT \\
\hline 6 & + & ++ & K642E & ++ & ++ & WT & WT \\
\hline 9 & \pm & + & V569G & ++ & ++ & WT & WT \\
\hline 10 & NA & - & W557R & NA & ++ & WT & WT \\
\hline 12 & ++ & + & WT & ++ & ++ & F66F & V600L \\
\hline 15 & NA & ++ & K642E & NA & ++ & WT & WT \\
\hline
\end{tabular}

Successful PCR amplification was obtained in 15 cases. (-), negative; $( \pm)$, focal; $(+)$, moderate; (++), intense; WT, wild-type; NA, not applicable.

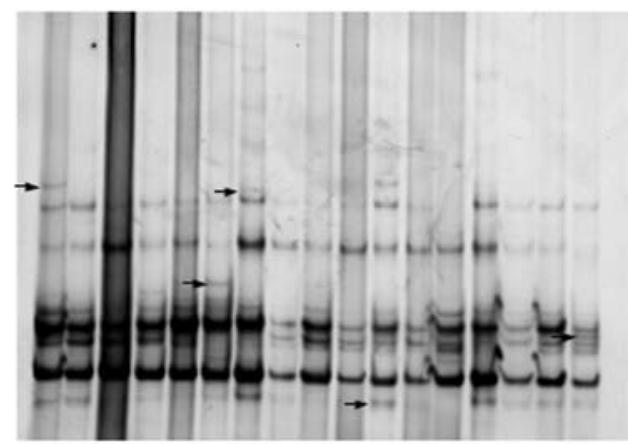

b
Normal sequence $106109 \quad 11211511 \varepsilon$

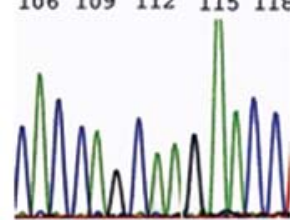

Lysine (K)
CAC CA G CA ISG AA C C
Mutated sequence CAC CAGCAAMAAC C $5109112 \overline{115} 118 \quad 1$ :
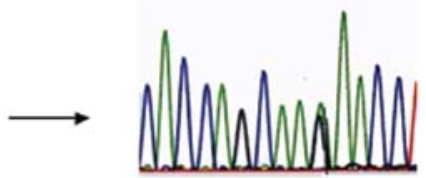

Lysine $(K)$

K166K
Figure 2. NRAS mutation. (a) Aberrant bands were observed in SSCP (arrows). (b) A point mutation was noted which led to the $\mathrm{G} \rightarrow \mathrm{A}$ change (b).

with PCR amplification (7\%) had a point mutation. Incidentally, the nucleotide $\mathrm{G} \rightarrow \mathrm{C}$ change altered the amino acid from a valine to a leucine (V600L) (Fig. 3b).

Results of RAS protein expression, and NRAS and BRAF mutations were compared to previously published c-kit results (Table III) (13). It was observed that NRAS and BRAF mutations are independent from c-kit mutations (cases 3 and 12). One NRAS silent mutation coincided with a missense mutation in BRAF wherein no c-kit mutation was found (case 12). Moreover, one RAS protein expression corresponded to a weak c-kit expression without a c-kit mutation (case 3). Furthermore, the case with BRAF and NRAS mutations had only moderate c-kit expression without a c-kit mutation (case 12). a

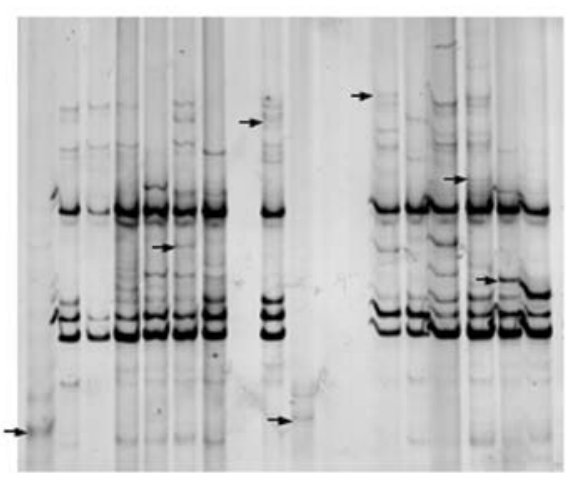

b

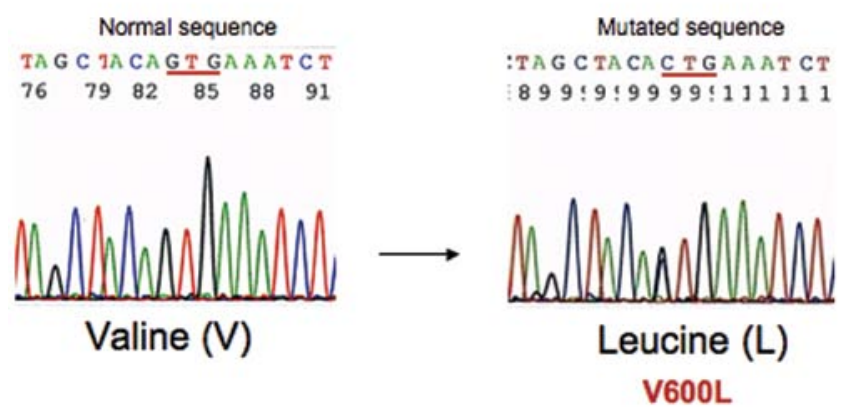

Figure 3. BRAF mutation. (a) Aberrant bands were observed in SSCP (arrows). (b) A point mutation was noted which led to the $\mathrm{G} \rightarrow \mathrm{C}$ change.

\section{Discussion}

With the breakthrough in the field of melanoma research, subsets of melanoma have been described based on anatomic site, sun exposure, histopathologic characteristics and genetic aberrations $(1,2,14,21)$. Investigations aim towards the identification of distinct genetic aberrations in order to target specific molecules responsible for the progression of the tumor. Nevertheless, the complexity behind melanoma oncogenesis is an impediment in improving the prognosis of this fatal type of cancer. Identification of molecular events specific to a subset of melanomas may help develop novel therapeutic agents. 
Mutation and up-regulation of c-kit have been studied in mucosal melanomas $(14,15,22,23)$. In particular, activating mutations have been correlated with increased c-kit protein expression (13). A mutated c-kit will lead to phosphorylation without binding of its ligand, stem cell factor, which in turn will activate downstream molecules. MAPK is the best-known pathway activated during the progression of melanoma. Wellknown downstream molecules include RAS, which activates BRAF.

A low frequency of NRAS and BRAF mutations was observed in the cases studied. The classical V600E mutation in BRAF was actually not found $(27,28)$. Instead, a novel V600L BRAF mutation was observed, which has not been reported in melanoma or in any other tumors. In spite of the lack in activating mutation in NRAS, RAS protein expression was intense in $89 \%$ of the cases. The intense RAS protein expression in both the in situ and invasive phases of OMM may suggest that RAS overexpression is necessary in OMM progression. Activating mutations in c-kit may have induced the intense NRAS protein expression (cases 6, 9, 10 and 15). These events could activate MAPK and PI3K pathways leading to cellular proliferation and survival.

It has been reported that NRAS and BRAF mutations are mutually exclusive $(15,19)$. Interestingly, the case with the BRAF missense mutation had wild-type c-kit (13) suggesting that the c-kit mutation is independent from the BRAF mutation. Other proteins, such as growth factors involved in OMM pathogenesis may activate NRAS or BRAF. Vascular endothelial growth factor (VEGF) and its receptor VEGFR-2 were expressed in OMM (24). Phosphorylation of VEGFR-2 by its ligand VEGF may activate RAS and RAF signal transduction cascades other than c-kit.

The frequency of intense NRAS protein expression, BRAF and c-kit activating mutations indicates that overlapping of molecular activities may occur in OMM progression posing a major concern in OMM therapy. The variation in genetic aberrations in melanoma as well as the lack of response in molecular therapies strongly supports the notion that OMM may have several putative oncogenic events. This might be another reason why immunotherapy targeting a single molecule was not sufficient. For instance, targeting V600E resulted in an initial response, but acquired resistance developed later (25). In this case, V600E mutation in BRAF may not be the sole genetic aberration in the patients not responding to the treatment. Furthermore, NRAS mutation or up-regulation has been observed in patients who developed acquired resistance to PLX4032 (26). Although NRAS and BRAF mutations are not that frequent in OMM, these molecules are still indispensable when considering immunotherapies in OMM. They may not be the prime factors, but they may be contributories to the oncogenic events in the progression of OMM.

A low frequency of NRAS and BRAF mutation is present in primary OMM. Nevertheless, RAS is intensely expressed in both in situ and invasive OMM. NRAS and BRAF mutations are not common, but probable events in OMM tumorigenesis, most likely independent of c-kit mutation. Overlapping of molecular events may occur in OMM progression, NRAS or BRAF activation may serve as alternative molecular mechanisms in OMM tumorigenesis.

\section{Acknowledgements}

The study was supported by grants-in-aid from the Japan Society for the Promotion of Science fellow (no. 22-00130), the Scientific Research (no. 21592326), and the Young Scientists (no. 22791766).

\section{References}

1. Barker BF, Carpenter WM, Daniels TE, et al: Oral mucosal melanomas: the WESTOP Banff workshop proceedings. Western Society of Teachers of Oral Pathology. Oral Surg Oral Med Oral Pathol Oral Radiol Endod 83: 672-679, 1997.

2. Hicks MJ and Flaitz CM: Oral mucosal melanoma: epidemiology and pathobiology. Oral Oncol 36: 152-169, 2000.

3. Nagatsuka H, Rivera RS, Gunduz M, et al: Immunolocalization and distribution patterns of type IV collagen alpha chains in oral mucosal melanoma. Virchows Arch 447: 710-716, 2005.

4. Buery RR, Siar CH, Katase N, et al: Clinico-pathological evaluation of oral melanotic macule, oral pigmented nevus and oral mucosal melanoma. J Hard Tis Biol 19: 57-64, 2010.

5. Satyamoorthy K, Li G, Gerrero MR, et al: Constitutive mitogenactivated protein kinase activation in melanoma is mediated by both BRAF and autocrine growth factor stimulation. Cancer Res 63: 756-759, 2003.

6. Sharma A, Tran MA, Liang S, et al: Targeting mitogen-activated protein kinase/extracellullar signal-regulated kinase kinase in the mutant (V600E) B-Raf-signaling cascade effectively inhibits melanoma lung metastases. Cancer Res 66: 82008209, 2006.

7. Smalley KS, Haass NK, Brafford PA, Lioni M, Flaherty KT and Herlyn M: Multiple signaling pathways must be targeted to overcome drug resistance in cell lines derived from melanoma metastases. Mol Cancer Ther 5: 1136-1144, 2006.

8. Solit DB, Garraway LA, Pratilas CA, et al: BRAF mutation predicts sensitivity to MEK inhibition. Nature 439: 358-362, 2006.

9. Wong CW, Fan YS, Chan TL, et al: BRAF and NRAS mutations are uncommon in melanomas arising in diverse internal organs. J Clin Pathol 58: 640-644, 2005.

10. Poynter JN, Elder JT, Fullen DR, et al: BRAF and NRAS mutations in melanoma and melanocytic nevi. Melanoma Res 16: 267-273, 2006.

11. Saldanha G, Potter L, Daforno P and Pringle JH: Cutaneous melanoma subtypes show different BRAF and NRAS mutation frequencies. Clin Cancer Res 12: 4499-4505, 2006.

12. Lennartsson J, Jelacic T, Linnekin D and Shivakrupa R: Normal and oncogenic forms of the receptor tyrosine kinase kit. Stem Cells 23: 16-43, 2005.

13. Rivera RS, Nagatsuka H, Gunduz M, et al: C-kit protein expression correlated with activating mutations in KIT gene in oral mucosal melanoma. Virchows Arch 452: 27-32, 2008.

14. Curtin JA, Busam K, Pinkel D and Bastian BC: Somatic activation of KIT in distinct subtypes of melanoma. J Clin Oncol 24: 4340-4346, 2006.

15. Beadling C, Jacobson-Dunlop E, Hodi FS, et al: KIT gene mutations and copy number in melanoma subtypes. Clin Cancer Res 14: 6821-6828, 2008

16. Edwards $\mathrm{RH}$, Ward MR, Wu H, et al: Absence of BRAF mutations in UV-protected mucosal melanomas. J Med Genet 41: 270-272, 2004

17. Maldonado JL, Fridlyand J, Patel H, et al: Determinants of BRAF mutations in primary melanomas. J Natl Cancer Inst 95: 1878-1879, 2003.

18. Cohen Y, Rosenbaum E, Begum S, et al: Exon 15 BRAF mutations are uncommon in melanomas arising in non-sun-exposed sites. Clin Cancer Res 10: 3444-3447, 2004.

19. Chernoff KA, Bordone L, Horst B, et al: GAB2 amplifications refine molecular classification of melanoma. Clin Cancer Res 15: 4288-4291, 2009.

20. Sekine S, Nakanishi Y, Ogawa R, Kouda S and Kanai Y: Esophageal melanomas harbor frequent NRAS mutations unlike melanomas of other mucosal sites. Virchows Arch 454: 513-517, 2009.

21. Curtin JA, Fridlyand J, Kageshita T, et al: Distinct sets of genetic alterations in melanoma. N Engl J Med 353: 2135-2147, 2005. 
22. Ashida A, Takata M, Murata H, Kido K and Saida T: Pathological activation of KIT in metastatic tumors of acral and mucosal melanomas. Int J Cancer 124: 862-868, 2009.

23. Satzger I, Schaefer T, Kuettler U, et al: Analysis of c-KIT expression and KIT gene mutation in human mucosal melanomas. Br J Cancer 99: 2065-2069, 2008.

24. Rivera RS, Nagatsuka $\mathrm{H}$, Siar $\mathrm{CH}$, et al: Heparanase and vascular endothelial growth factor expression in the progression of oral mucosal melanoma. Oncol Rep 19: 657-661, 2008.

25. Flaherty KT, Puzanov I, Kim KB, et al: Inhibition of mutated, activated BRAF in metastatic melanoma. N Engl J Med 363: 809-819, 2010.
26. Nazarian R, Shi H, Wang Q, et al: Melanomas acquire resistance to BRAF (V600E) inhibition by RTK or NRAS upregulation. Nature 468: 973-977, 2010

27. Davies H, Bignell GR, Cox C, et al: Mutations of the BRAF gene in human cancer. Nature 417: 949-954, 2002.

28. Wan PT, Garnett MJ, Roe SM, et al: Mechanism of activation of the RAF-ERK signaling pathway by oncogenic mutations of BRAF. Cell 116: 855-867, 2004. 